\title{
Generation and characterisation of therapeutic tolerogenic dendritic cells for rheumatoid arthritis
}

\author{
Rachel A Harry, Amy E Anderson, John D Isaacs, Catharien M U Hilkens
}

- Additional data are published online only. To view these files please visit the journal online (http://ard.bmj.com).

Institute of Cellular Medicine, Musculoskeletal Research Group, Newcastle University, Newcastle Upon Tyne, UK

\section{Correspondence to \\ Dr Catharien Hilkens, Institute of Cellular Medicine, Musculoskeletal Research Group, Newcastle University, Framlington Place, Newcastle Upon Tyne NE2 4HH, UK; catharien.hilkens@newcastle. ac.uk}

Accepted 5 April 2010

\section{(6) UNLOCKED}

This paper is freely available online under the BMJ Journals unlocked scheme, see http:// ard.bmj.com/info/unlocked.dtl

\section{ABSTRACT}

Objectives Tolerogenic dendritic cells (tolDCs) constitute a promising experimental treatment for targeting autoreactive T cells in autoimmune diseases, including rheumatoid arthritis (RA). The authors' goal is to bring tolDC therapy for RA to the clinic. Here the authors address key translational issues related to the manufacturing of tolDCs from RA patients with current good manufacturing practice (cGMP)-compliant reagents, the stability of toIDCs, and the selection of suitable quality control markers.

Methods Human monocyte-derived tolDCs were established from RA patients and healthy controls (HCs) using the immunosuppressive drugs dexamethasone and vitamin $\mathrm{D}_{3}$ and the cGMP-grade immunomodulator, monophosphoryl lipid $\mathrm{A}$, in the cGMP-compliant medium, CellGroDC. The functionality of tolDCs and tolDCmodulated autologous CD4 T cells was determined by flow cytometry, $\left[{ }^{3} \mathrm{H}\right]$ thymidine incorporation and ELISA.

Results Clinical-grade tolDCs established from patients with RA exhibit a typical tolerogenic phenotype of reduced costimulatory molecules, low production of proinflammatory cytokines and impaired stimulation of autologous antigen-specific T cells, comparable to $\mathrm{HC}$ tolDCs. Toll-like receptor 2 (TLR-2) was highly expressed by tolDCs but not mature DCs. Furthermore, tolDCs suppressed mature DC-induced T cell proliferation, interferon $\gamma$ and interleukin 17 production, and rendered T cells hyporesponsive to further stimulation. Importantly, tolDCs were phenotypically stable in the absence of immunosuppressive drugs and were refractory to further challenge with proinflammatory mediators.

Conclusions tolDCs established from patients with RA are comparable to those derived from healthy donors. TLR-2 was identified as an ideal marker for quality control of tolDCs. Potently tolerogenic and highly stable, these toIDCs are a promising cellular therapeutic for tailored immunomodulation in the treatment of RA.

\section{INTRODUCTION}

Rheumatoid arthritis (RA) is a chronic, debilitating autoimmune disease with no known cure. Autoimmune diseases including RA are thought to arise through a breakdown in self-tolerance. Current treatments involve non-antigen-specific global immunosuppression leading to numerous side effects. Much research has therefore focused on the development of more selective immunosuppressive, drug-sparing therapies with fewer complications and the potential of long-term disease remission. T cell immunomodulation, or immune reprogramming, is an attractive strategy for treatment of autoimmune disorders, ${ }^{1}$ and a novel approach for targeting autoreactive $T$ cells is the use of antigen-specific dendritic cells (DCs). ${ }^{2}$
DCs are critical in the initiation of immune responses against invading pathogens and tumours, but also in the induction of central and peripheral tolerance to self-antigens. ${ }^{3}$ The constitutive depletion of DCs in mice leads to a collapse in self-tolerance and the development of fatal autoimmune disease. ${ }^{4}$ Tolerogenic DCs (tolDCs) induce tolerance through the presentation of antigen with inadequate costimulation and cytokine production for effector $\mathrm{T}$ cell activation, resulting in $\mathrm{T}$ cell silencing or deletion or induction of regulatory T cells. ${ }^{56}$

tolDCs can be generated in vitro by a variety of methods, including genetic or pharmacological modification. ${ }^{7}$ Injection of ex vivo modified tolDCs has proven beneficial in models of autoimmune disease, including collagen-induced arthritis, ${ }^{8-18}$ diabetes, ${ }^{19}$ experimental autoimmune encephalomyelitis ${ }^{16}$ and uveoretinitis. ${ }^{20}$ Therapy with tolDCs therefore has great potential for the treatment of autoimmunity in humans, and the current challenge is to develop tolDCs for clinical application. Others have addressed the development of clinical-grade DCs for cancer immunotherapy. ${ }^{21-23}$ However, criteria for the development of safe tolDCs for the treatment of autoimmune disease remain to be evaluated.

Previously, we have described a simple and robust method for the establishment of tolDCs by treatment of monocyte-derived DCs with the immunosuppressive glucocorticoid, dexamethasone (Dex), the vitamin $\mathrm{D}$ receptor agonist, $1,25(\mathrm{OH})_{2} \mathrm{D}_{3}$ (vitamin $\mathrm{D}_{3}\left(\mathrm{VitD}_{3}\right)$ ) and the toll-like receptor 4 (TLR-4) ligand, lipopolysaccharide (LPS) from Escherichia coli. 2425 These tolDCs, characterised by low expression of costimulatory molecules and low production of proinflammatory cytokines, have impaired $T$ cell stimulatory capacity and tolerise T cells in vitro. Our aim is to conduct clinical trials with these tolDCs for RA. ${ }^{2627}$

Here we address several key translational issues pertaining to clinical application of tolDCs. Firstly, research-grade reagents-for example, fetal bovine serum or LPS-need to be substituted for current good manufacturing practice (cGMP)-compatible reagents, without compromising the $\mathrm{T}$ cell-tolerising effects of tolDCs. Furthermore, monocyte-derived DCs are more proinflammatory in RA, ${ }^{28} 29$ therefore our protocol requires validation to guarantee generation of effective tolDCs from patients with RA. Another important consideration is the selection of reliable and easy-to-measure quality control (OC) markers. OC markers should ideally be highly expressed, ${ }^{30} 31$ exclusively by tolDCs, rather than reliant on comparative expression with mature immunogenic DCs. Finally, as tolDCs will 
be used to treat RA, a chronic inflammatory disease, their stability in proinflammatory environments is paramount and requires investigation. This study describes a cGMP-compatible method for generating stable tolDCs from patients with RA, resulting in a novel autologous cellular therapy for the specific modulation of autoreactive $\mathrm{T}$ cells for the treatment of RA.

\section{MATERIALS AND METHODS}

\section{Ethics}

Peripheral blood samples from healthy controls (HCs) and patients with RA were obtained with informed consent and after approval by the Newcastle and North Tyneside Research Ethics Committee 2.

\section{Isolation of cells}

Peripheral blood mononuclear cells were isolated by density centrifugation. Monocytes were positively selected from peripheral blood mononuclear cells using anti-CD14 microbeads (Miltenyi Biotec, Bergisch Gladbach, Germany). CD4 T cells were enriched using CD4 RosetteSep (StemCell, Vancouver, BC, Canada) or by positive selection with anti-CD4 microbeads (Miltenyi Biotec).

\section{Establishment of DCs}

Monocytes were cultured at $0.5 \times 10^{6} / \mathrm{ml}$ for 7 days in cGMP-grade CellGroDC (CellGenix, Freiburg, Germany) containing penicillin and streptomycin solution $(100 \mathrm{U} / \mathrm{ml}$ and $100 \mu \mathrm{g} / \mathrm{ml}$, respectively) in the presence of clinical-grade granulocyte-macrophage colony-stimulating factor $(50 \mathrm{ng} / \mathrm{ml}$; Berlex, Seattle, Washington, USA) and interleukin 4 (IL-4; 50 ng/ml; Immunotools, Friesoythe, Germany). Cells were replenished on day 3 with fresh medium and cytokines, and cytokines only on day 6. To induce mature DCs (matDCs), DCs were treated with cGMP-grade monophosphoryl lipid A (MPLA) $(1.0 \mu \mathrm{g} / \mathrm{ml}$; Avanti Polar Lipids, Alabaster, Alabama, USA) on day 6. tolDCs were established by treatment with Dex (1 $\mu \mathrm{M}$, Sigma Aldrich, Poole, UK) on day 3 and Dex $(1 \mu \mathrm{M})$ and $\mathrm{VitD}_{3}(0.1 \mathrm{nM}$, Biomol, Exeter, UK) and MPLA on day 6 . On day 7, DCs were harvested and washed extensively before functional assays were performed. DCs were loaded during the final $24 \mathrm{~h}$ of culture with the recall protein antigen, purified protein derivative (PPD; $1 \mu \mathrm{g} / \mathrm{ml}$; Statens Serum Institut, Copenhagen, Denmark), or candidate RA autoantigen human cartilage glycoprotein 39 (HCgp39; $10 \mu \mathrm{g} / \mathrm{ml}$; Organon, Welwyn Garden City, UK). Control DCs were left unloaded. For superantigen experiments, DCs were pulsed with $5 \mathrm{ng} / \mathrm{ml}$ toxic shock syndrome toxin 1 (TSST-1) for $1 \mathrm{~h}$.

\section{DC phenotyping}

Cell surface expression was investigated using the following antibodies: CD14-PE (M5E2), CD40-APC (5C3), CD80-FITC (L307.4), CD86-APC (FUN-1), CD83-PE (HB15e), HLA-DR DP DQ-FITC (Tu39), all from BD Biosciences (Oxford, UK), and TLR-2-APC (TL2.1; e-Bioscience, Hatfield, UK). DCs were washed and resuspended in staining buffer (phosphate-buffered saline supplemented with $1 \%$ fetal calf serum, 2 mM EDTA and $0.01 \%$ sodium azide) in the presence of IgG (Grifols, Los Angeles, California, USA), and incubated with antibody for 30 min before acquisition on a BD FACScan and analysed using FlowJo software (Treestar).

\section{DC cytokine production}

DCs were stimulated at $1 \times 10^{5} \mathrm{DCs} / \mathrm{ml}$ with CD40-ligandtransfected J558L mouse cells (a gift from Professor Peter
Lane, Birmingham University, UK) at a 1:1 ratio for $24 \mathrm{~h}$. Cytokine production was determined in supernatants by sandwich ELISA from BD Pharmingen (Oxford, UK) (IL-6, IL-10, IL-12p70, tumour necrosis factor (TNF) $\alpha$ ) or e-Bioscience (IL-1 $\beta$, IL-23).

\section{DC/T cell cultures}

$\mathrm{DC} / \mathrm{T}$ cell cultures were carried out in complete medium: RPMI1640 containing human $\mathrm{AB}^{+}$serum $(5 \%)$, L-glutamine (2 mM), and penicillin $(100 \mathrm{U} / \mathrm{ml})$ and streptomycin $(100 \mu \mathrm{g} / \mathrm{ml})$. T cells $\left(10^{5}\right)$ were cultured with unloaded or antigen-loaded tolDCs or matDCs. In restimulation experiments, primed $\mathrm{T}$ cells were subsequently rested from day 6 to 10 in the presence of $20 \mathrm{IU} / \mathrm{ml}$ recombinant IL-2 (Novartis, Camberley, UK) and restimulated on day 10 with antigen-loaded autologous matDCs for 3 days. $\mathrm{DC} / \mathrm{T}$ cell cultures were performed at varying ratios. Proliferation was determined by incorporation of $\left[{ }^{3} \mathrm{H}\right]$ thymidine for $18 \mathrm{~h}$ or by carboxyfluorescein succinimidyl ester $(0.5 \mu \mathrm{M})$ dilution analysis of $T$ cell receptor $\beta$-chain variable region 2 (V $\beta 2$ )-expressing cells (anti-TCR-V $\beta 2$; clone MPB2D5; Immunotech, Marseille, France) at various time points. For the tolDC-suppression assay, additional tolDCs or matDCs were incorporated as indicated. Cytokine production in $\mathrm{T}$ cell supernatants was determined by sandwich ELISA for interferon $\gamma$ (IFN $\gamma$ ) (BD Pharmingen) and IL-17 (e-Bioscience).

\section{Intracellular cytokine staining}

$T$ cells restimulated with matDCs for 3 days were activated for maximal cytokine production with phorbol myristate acetate $(50 \mathrm{ng} / \mathrm{ml})$ and ionomycin $(1 \mu \mathrm{g} / \mathrm{ml})$ for $5 \mathrm{~h}$ in the presence of brefeldin A $(10 \mu \mathrm{g} / \mathrm{ml})$ for the final $4 \mathrm{~h}$. Cells were stained for cell surface V $\beta 2$ expression before fixation and permeabilisation (FoxP3 staining kit; e-Bioscience) and subsequent staining for intracellular IL-17 (clone eBio64DEC17) and IFNy (clone B27). Cells were acquired on a BD LSRII and analysed using FlowJo software.

\section{DC stability}

Phenotypic stability of DCs was investigated $24 \mathrm{~h}$ after washing and reculture of DCs in complete medium. Firstly, $1 \times 10^{6}$ cells/ $\mathrm{ml}$ were left unstimulated or stimulated with a cocktail of proinflammatory cytokines containing $1000 \mathrm{U} / \mathrm{ml} \mathrm{IFN \gamma}$ and $10 \mathrm{ng} /$ $\mathrm{ml}$ each of IL-1 $\beta$, IL-6 and TNF $\alpha, 0.1 \mu \mathrm{g} / \mathrm{ml} \mathrm{LPS} \mathrm{or} 10 \mu \mathrm{g} / \mathrm{ml}$ peptidoglycan (PGN). Cells were stained as described for DC phenotyping, and cytokine production was investigated in the absence and presence of proinflammatory cytokines, LPS or PGN as described for DC cytokine production.

\section{Statistical analysis}

The following statistical analyses were performed using Prism 4.0: analysis of variance (ANOVA) for comparisons between multiple groups, Mann-Whitney U or Student t test for comparisons between two groups, and two-way ANOVA for multiple groups over time.

\section{RESULTS \\ cGMP-tolDCs from patients with RA are phenotypically and functionally comparable to HC tolDCs}

Our initial studies investigated the surface phenotype, cytokine profile and $\mathrm{T}$ cell-stimulatory capacity of tolDCs from RA patients as compared with HCs, established using the cGMPmedium CellGroDC and bacterial immunomodulator MPLA. 
A
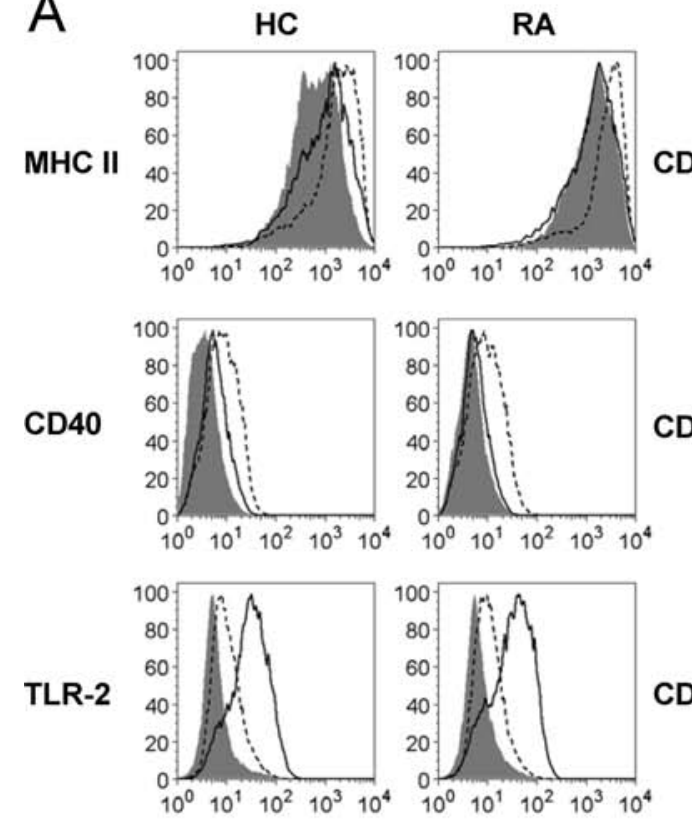
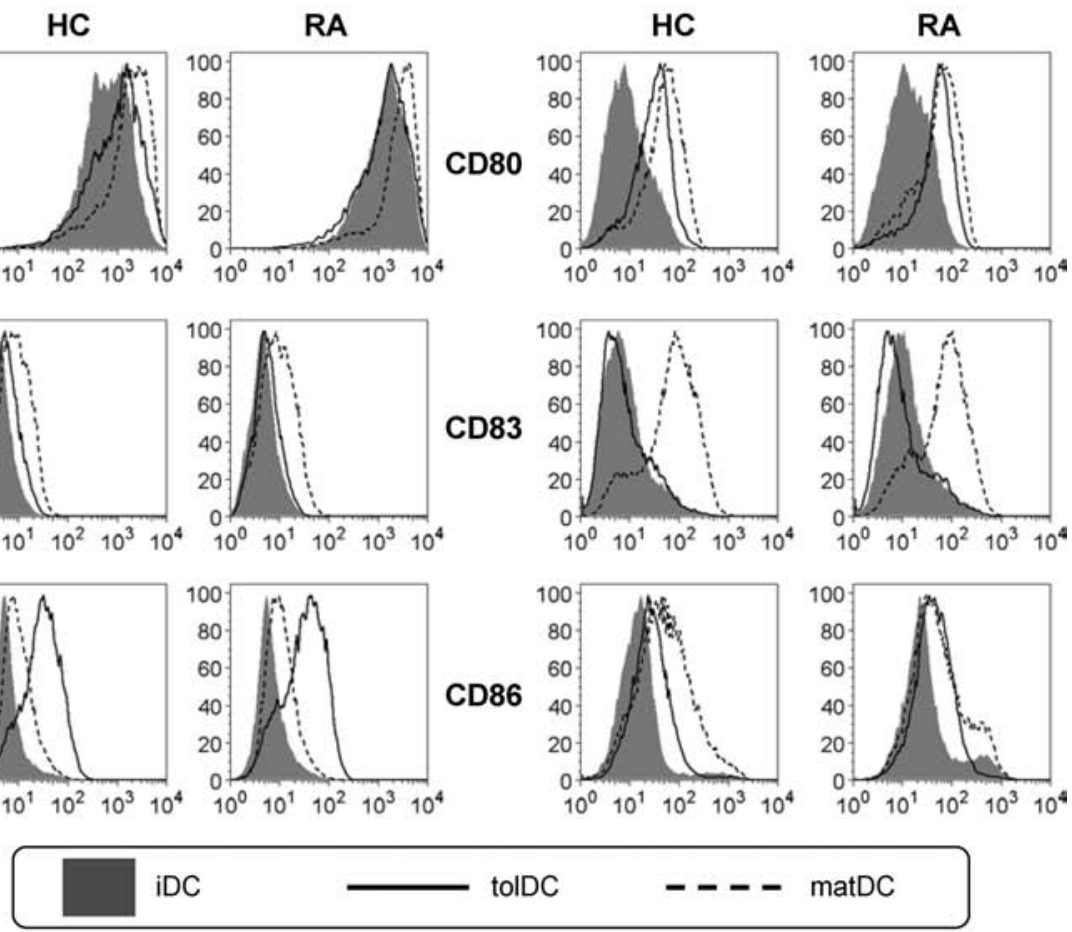

B

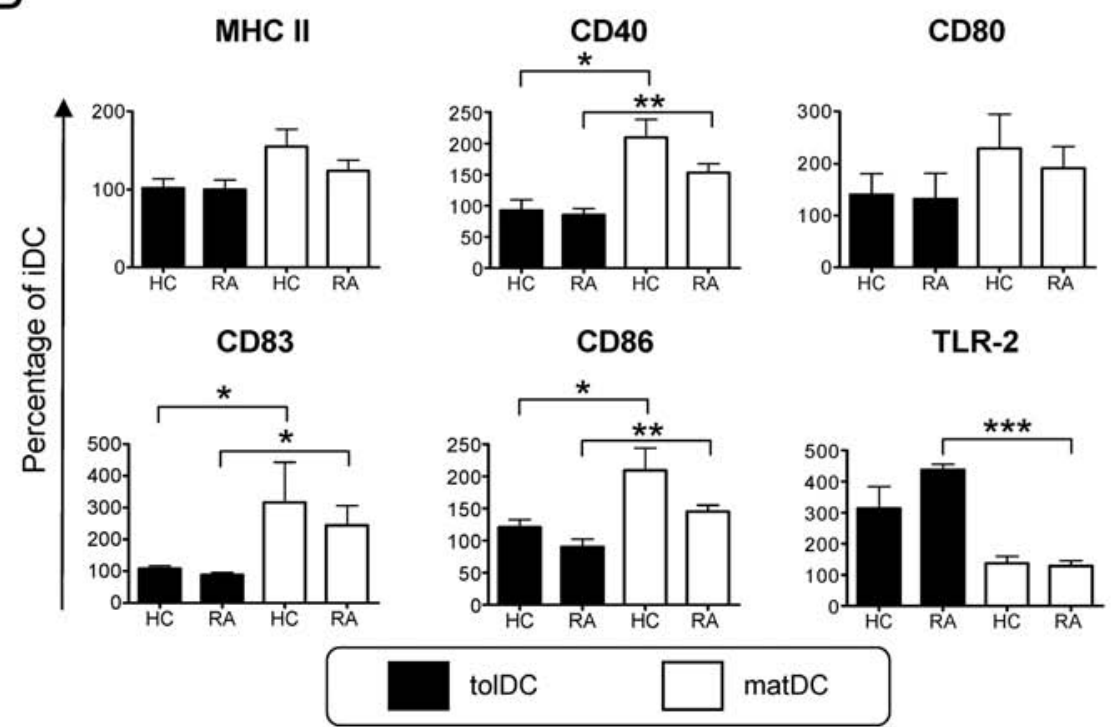

Figure 1 Rheumatoid arthritis (RA)-derived tolerogenic dendritic cells (tolDCs) exhibit a semimature phenotype and are comparable to healthy control (HC) tolDCs. (A) HC- and RA-derived DC expression of maturation-associated markers of immature DCs (iDCs), mature DCs (matDCs) and tolDCs. Histograms are representative of five independent donors. (B) Mean expression of HC and RA patient tolDCs and matDCs is shown relative to iDCs. Expression of each marker (geometric mean fluorescence intensity (GMFI)) was normalised relative to expression by iDCs (100\%) for each independent donor. Phenotypic analysis of maturation-associated markers was determined $24 \mathrm{~h}$ after maturation with $1 \mu \mathrm{g} / \mathrm{ml}$ monophosphoryl lipid $A$ in the presence or absence of dexamethasone and vitamin $D_{3}$. Data are expressed as the mean $\pm S E M$ for five independent donors. Mean GMFI for $\mathrm{HC}$ iDCs vs RA iDCs are: MHC II, $949.8 \pm 161.3$ vs $1110 \pm 178.3$; CD40, 19.8 \pm 7.4 vs $20.4 \pm 6.6$; CD80, $12.3 \pm 1.7$ vs $11.2 \pm 1.4$; CD83, $7.9 \pm 0.1$ vs $9.4 \pm 1.2$; CD86, $11.5 \pm 2.5$ vs $21.6 \pm 5.8$; toll-like receptor 2 (TLR-2), $89.9 \pm 11.1$ vs $76.2 \pm 4.2$. No significant difference was detected between RA and HC DC populations. ${ }^{*} \mathrm{p} \leq 0.05,{ }^{* *} \mathrm{p} \leq 0.01,{ }^{* * *} \mathrm{p} \leq 0.001$ (analysis of variance). MHC, major histocompatibility complex; TLR-2, toll-like receptor-2.

Immature DCs (iDCs; figure $1 \mathrm{~A}$ ) expressed reduced levels of major histocompatibility complex II (MHC II) and low levels of CD40, CD83 and the costimulatory molecules CD80 and CD86. Expression of all these markers was substantially enhanced upon DC maturation (matDCs; figure $1 \mathrm{~A}, \mathrm{~B}$ ). In contrast, tolDCs from both RA patients and HCs displayed a typical semimature phenotype of reduced MHC II and CD80 and significantly lower CD40, CD83 and CD86 expression than
matDCs (figure 1B). A highly expressed marker on tolDCs was TLR-2, which was low on iDCs and matDCs (figure 1A,B) and may therefore be suitable for $\mathrm{OC}$. Despite the high propensity of RA-matDCs to produce TNF $\alpha$, production of all other cytokines investigated was comparable between RA and $\mathrm{HC}$ cultures (figure 2A). tolDCs from both RA patients and HCs consistently produced lower levels of proinflammatory cytokines IL-1 $\beta$, IL- 6 , IL-23 and TNF $\alpha$ upon CD40-mediated 
activation, as compared with matDCs, while IL-12 was notably undetected in all tolDC cultures (figure 2A). Similar differences were found when cytokine production by tolDCs and matDCs was measured directly after maturation with MPLA, before activation through CD40 (online supplemental figure 1). Anti-inflammatory IL-10 production by tolDCs was non-significantly reduced as compared with their equivalent matDC counterparts.

The cytokine profile of tolDCs was unaffected by incorporation of an antigen-loading step during tolDC generation, investigated using a RA candidate autoantigen (HCgp39; figure 2B). Furthermore, we investigated the autologous $\mathrm{T}$ cell immunostimulatory capacity of tolDCs using the recall antigen, PPD. Minimal T cell proliferation was observed in response to both RA and $\mathrm{HC}$ non-antigen-loaded DC populations, while PPD-loaded matDCs induced significant $T$ cell proliferation. Consistent with their semimature phenotype and anti-inflammatory cytokine profile, both RA and HC PPD-loaded tolDCs only poorly induced antigen-specific proliferation (figure $3 \mathrm{~A}, \mathrm{~B}$ ) and IFNy and IL-17 production by autologous $\mathrm{T}$ cells (figure $3 \mathrm{~B}$ ).

\section{tolDCs induce $\mathrm{T}$ cell hyporesponsiveness}

We further investigated the immunoregulatory capacity of clinical-grade tolDCs in a superantigen-based $\mathrm{T}$ cell activation model. This model is well established for studying $T$ cell activation and modulation by autologous DCs. ${ }^{32-36}$ TSST-1 binds with high affinity to TCR-V $\beta 2$, preferentially expanding V $\beta 2^{+}$ $\mathrm{T}$ cells in a MHC II-dependent manner, ${ }^{37}$ although expansion of low-affinity V $\beta 2^{-} \mathrm{T}$ cells can occur. ${ }^{32}$ Here we quantitated the $T$ cell response to tolDCs and matDCs loaded with TSST-1 (figure 4A). matDCs were superior over tolDCs in inducing $\mathrm{T}$ cell proliferation, inducing activation of $\mathrm{V} \beta 2^{+} \mathrm{T}$ cells even at

A

IL-1及

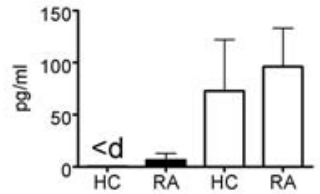

IL-6

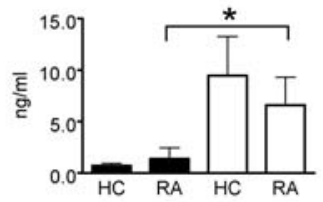

IL-10

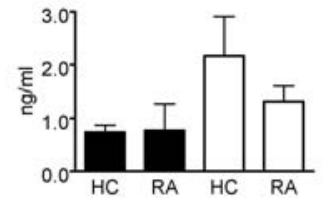

IL-12p70

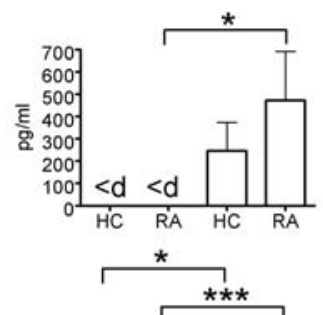

IL-23
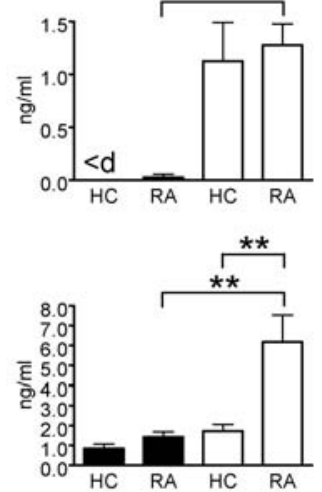

Figure 2 Healthy control $(\mathrm{HC})$ and rheumatoid arthritis (RA) tolerogenic dendritic cells (tolDCs) have an anti-inflammatory phenotpye. (A) Mean cytokine production by tolDCs and mature DCs (matDCs) in response to CD40 activation. At $24 \mathrm{~h}$ after maturation with $1 \mu \mathrm{g} / \mathrm{ml}$ monophosphoryl lipid $A(M P L A)$ in the presence or absence of dexamethasone (Dex) and vitamin $D_{3}\left(V_{i t}\right)$ DCs were washed, and $10^{5}$ tolDCs or matDCs cocultured at a 1:1 ratio with the $\mathrm{J588 \textrm {L } - C D 4 0 \mathrm { L } - e x p r e s s i n g ~ c e l l ~ l i n e ~ i n ~ t h e ~ a b s e n c e ~ o f ~ D e x ~ a n d ~ V i t D ~}{ }_{3}$ for a further $24 \mathrm{~h}$. Supernatants were harvested for cytokine analysis by ELISA. Data are expressed as the mean \pm SEM for at least four HCs and seven RA-independent donors. (B) Representative RA donor DC-derived cytokine production when unloaded (UL) or loaded with $10 \mu \mathrm{g} / \mathrm{ml}$ human cartilage glycoprotein 39 (HCgp39) as a model antigen, during maturation with $1 \mu \mathrm{g} / \mathrm{ml}$ MPLA. Unloaded and antigen-loaded DCs were activated via CD40 as described in (A). Data are representative of four independent donors. Cytokine production was determined by ELISA. $<$ d, below limits of detection. Detection limit for IL-12 is $30 \mathrm{pg} / \mathrm{ml}$. ${ }^{*} \mathrm{p} \leq 0.05$, ${ }^{* *} \mathrm{p} \leq 0.01,{ }^{* *} \mathrm{p} \leq 0.001$ (Mann-Whitney U test). TNF, tumour necrosis factor. 


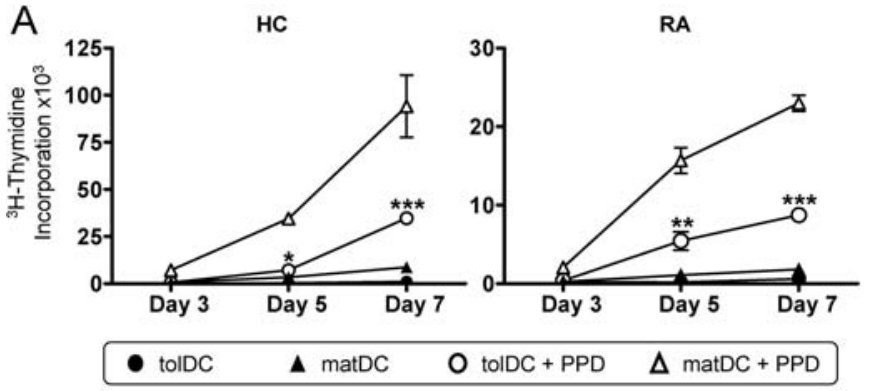

B

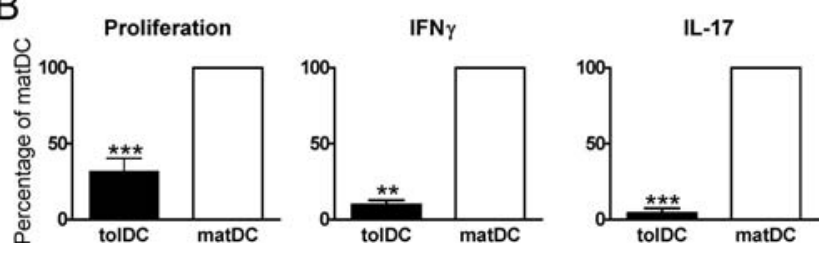

Figure 3 Antigen-specific T cells exhibit a reduced responsiveness to tolerogenic dendritic cells (tolDCs). (A) $10^{5}$ healthy control (HC) and RA CD4 T cells were stimulated with $10^{4}$ autologous unloaded or purified protein derivative (PPD)-loaded $(1 \mu \mathrm{g} / \mathrm{ml}$ ) tolDCs or mature DCs (matDCs), and proliferation determined at various time points. Data are expressed as the mean \pm SEM of triplicate wells, representative of three independent experiments. (B) Mean healthy control (HC) CD4 T cell proliferation and cytokine production as a percentage of matDCinduced T cell responses. HC CD4 T cells $\left(10^{5}\right)$ were stimulated at a 10:1 ratio with $10^{4} \mathrm{PPD}$-loaded tolDCs or matDCs. Cytokine production and proliferation were determined on day 6 and 7, respectively. Data are expressed as the mean of three independent experiments. Mean values for matDC-induced proliferation were $33642 \pm 18807$ $\mathrm{cpm}$, interferon (IFN) $\gamma$ was $16.9 \pm 8.1 \mathrm{ng} / \mathrm{ml}$ and interleukin (IL)-17 was $359.5 \pm 328.8 \mathrm{pg} / \mathrm{ml}$. T cell proliferation was determined by $\left[{ }^{3} \mathrm{H}\right]$ thymidine incorporation, and cytokine production was quantitated by ELISA. ${ }^{*} p \leq 0.05,{ }^{* *} p \leq 0.01,{ }^{* *} p \leq 0.001$ as compared with matDCs (two-way analysis of variance $(A)$ and Student $t$ test $(B)$ ).

very low DC numbers and also inducing expansion of $\mathrm{V} \beta 2^{-} \mathrm{T}$ cells at higher DC numbers. In contrast, tolDCs expanded $\mathrm{V} \beta 2^{+}$ $T$ cells at high DC concentrations, but failed to support strong $\mathrm{V} \beta 2^{-} \mathrm{T}$ cell proliferation (figure $4 \mathrm{~A}$ ). Upon optimal restimulation with TSST-1-loaded matDCs, T cells primed by TSST-1loaded tolDCs (Ttol) showed impaired proliferative potential (figure $4 \mathrm{~B}$ ) and a reduction in the percentage of IFN $\gamma$ - and IL-17-producing $\mathrm{T}$ cells as compared with $\mathrm{T}$ cells primed by TSST-1-loaded matDCs (Tmat) (figure 4C), suggestive of Ttol hyporesponsiveness.

\section{tolDCs suppress matDC-induced $\mathrm{T}$ cell proliferation, IFN $\gamma$ and IL-17}

Successful tolDC therapy may in part depend on the ability to suppress immune responses induced by resident immunogenic DCs. We therefore investigated the ability of tolDCs to modulate matDC-induced PPD-specific $T$ cell proliferation and cytokine production. Co-incubation of PPD-loaded tolDCs and matDCs significantly inhibited $T$ cell proliferation (figure $5 \mathrm{~A}$ ), IFN $\gamma$ and IL-17 production (figure $5 \mathrm{~B}$ ) at a 1:1 tolDC/matDC ratio and significantly inhibited proliferation (figure $5 \mathrm{~A}$ ) and IFN $\gamma$ production at a $1: 10$ tolDC/matDC ratio (figure $5 \mathrm{~B}$ ) as compared with PPD-loaded matDCs alone. These inhibitory effects were not caused by increasing the total DC number, as doubling the number of matDCs did not result in suppression of $T$ cell proliferation and even enhanced cytokine production (figure 5A,B).
toIDCs have a highly stable semimature, anti-inflammatory phenotype

A key consideration in the development of a tolDC therapy for RA and other chronic inflammatory diseases is the stability of tolDCs to proinflammatory mediators. tolDC stability was therefore investigated after removal of Dex, $\mathrm{VitD}_{3}$ and MPLA and reculture in medium containing human serum. Both tolDCs and matDCs were phenotypically refractory to stimulation with all proinflammatory mediators investigated, with tolDCs retaining their typical semimature phenotype and cytokine profile (figure 6A,B). TLR-2 expression by tolDCs was found to be downregulated comparable to that of matDC expression (figure 6A), while the only marker that was significantly upregulated by tolDCs was CD83 in response to proinflammatory cytokines, although levels remained significantly $(p<0.01$; paired $t$ test) lower than that expressed by equivalent matDCs (figure 6A). CD40-activation-induced cytokine production by tolDCs and matDCs was unaltered in response to cytokine or LPS challenge (figure 6B), while PGN induced increases in IL-10 production by both tolDCs and matDCs. IL-12p70 remained undetectable in tolDC cultures under all conditions investigated (figure 6B). Overall, these data indicate that tolDCs are highly stable.

\section{DISCUSSION}

This study describes the development of clinical-grade tolDCs, focusing on a number of key translational issues that require elucidation before therapeutic application of these cells.

We investigated the functionality of tolDCs generated using cGMP-grade reagents, suitable for the development of an Investigational Medical Product for human therapy. We describe a protocol for the establishment of semimature, antiinflammatory, clinical-grade tolDCs using the cGMP-compliant serum-free medium CellGroDC and DC immunomodulator, MPLA. These clinical-grade tolDCs are comparable to our previously characterised research-grade tolDCs ${ }^{24} 25$ in terms of cell surface phenotype, cytokine production and in vitro $\mathrm{T}$ cell modulatory function. CellGroDC was deemed the medium of choice $^{23}$ and is currently used in DC vaccine development for cancer. ${ }^{38-40}$ MPLA, a low-toxicity alternative to LPS, is currently being tested in vaccine development. ${ }^{41}$ As a known TLR-4 agonist inducing matDCs, ${ }^{42}$ MPLA is less potent than LPS, ${ }^{43}$ which may explain the increased MPLA dose $(1.0 \mu \mathrm{g} / \mathrm{ml}$ MPLA vs 0.1 $\mu \mathrm{g} / \mathrm{ml}$ LPS $^{25}$ ) required to induce comparable DC maturation. ${ }^{42-44}$ We observed that MPLA potently induces IL-10 production by DCs. Considering the inhibitory effect of IL-10 on IL-12p35 and p40 mRNA $^{45} 46$ and IL-12p40 protein, ${ }^{46}$ it is not surprising that MPLA only poorly induced IL-12p70 and IL-23 (consisting of IL-12p40 and IL-23p19) by matDCs in this study. As the lack of IL-12 production by tolDCs is pivotal to their tolerogenicity, ${ }^{24} 47$ MPLA by virtue of its inability to induce IL-12 is highly appropriate for the establishment of tolDCs, but less suitable for the generation of matDC therapy for cancer.

As antigen-presenting cell dysregulation has been implicated in RA pathogenesis, ${ }^{48}$ we confirmed that this protocol could be used for the establishment of tolDCs from patients with RA. Indeed, in our and other studies, ${ }^{28} 29$ matDCs established from patients with active RA produced significantly more TNF $\alpha$ than $\mathrm{HCs}$ or RA patients in remission. We demonstrate that, despite an inherently more proinflammatory cytokine profile, tolDCs established from RA patients adopt a HC-tolDC-like phenotype, suggesting that, even with potentially altered antigen-presenting cell functionality during RA, it remains possible to establish 
A

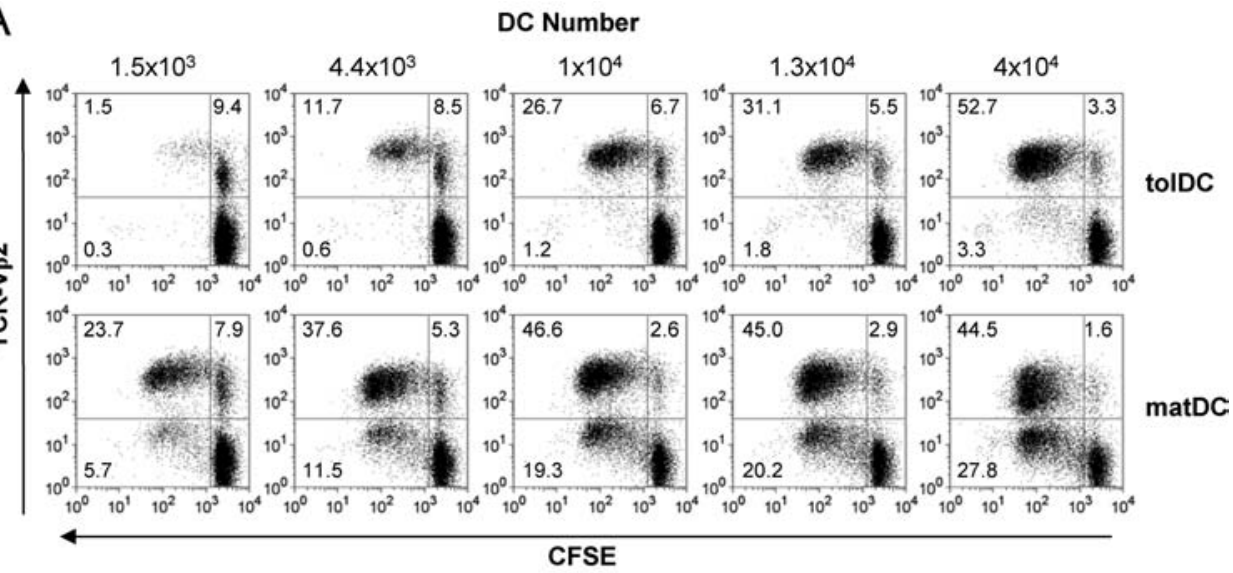

B

C
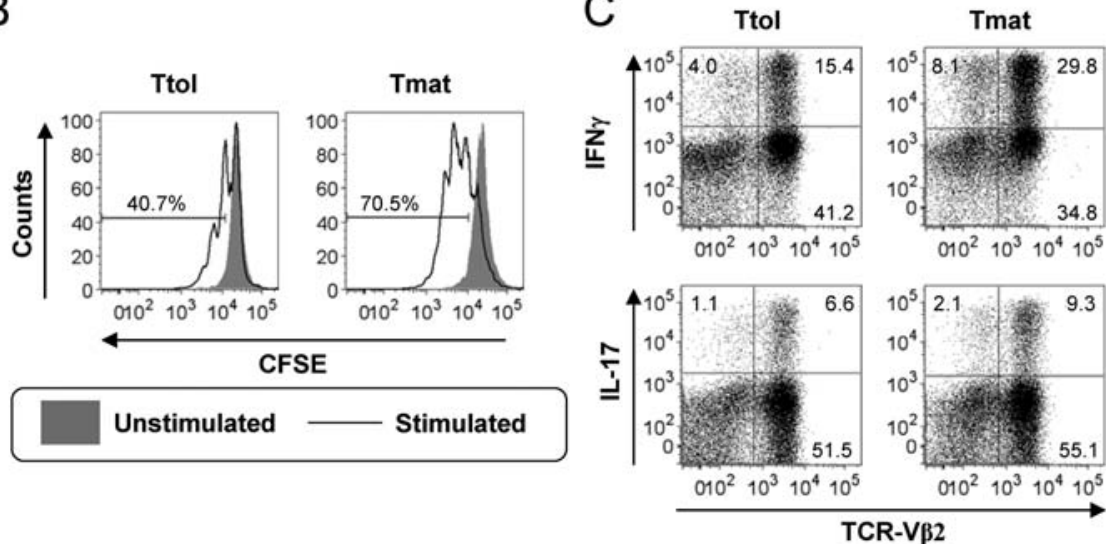

Figure 4 Tolerogenic dendritic cells (tolDCs) induce T cell hyporesponsiveness. (A) $10^{5}$ CD4 T cells were primed with the indicated numbers of toxic shock syndrome toxin 1 (TSST-1)-loaded ( $5 \mathrm{ng} / \mathrm{ml}$ ) autologous tolDCs or mature DCs (matDCs) and proliferation of carboxyfluorescein succinimidyl ester $\left(\mathrm{CFSE}^{+}\right.$) T cell receptor $\beta$-chain variable region 2 (TCR-V $\beta 2$ )-stained cells quantitated at day 5 . Values represent percentage of cells as a percentage of CD4 T cell population. Percentage of V $32^{+}$cells in starting CD4 population was $8.9 \pm 0.95 \%$. (B) and (C) T cells primed with TSST-1loaded ( $5 \mathrm{ng} / \mathrm{ml}$ ) tolDCs (Ttol) or matDCs (Tmat) (10:1 ratio) for 6 days were rested with interleukin (IL)-2 (20 IU/ml) for 4 days before CFSE labelling and restimulation at a 10:1 ratio with TSST-1-loaded matDCs on day 10. (B) Day 13 T cell proliferation was determined by CFSE dilution. Values represent the percentage of proliferating cells. (C) Day 13 intracellular IL-17 and interferon (IFN) $\gamma$ production was quantitated after $5 \mathrm{~h}$ stimulation with phorbol myristate acetate (PMA) $(50 \mathrm{ng} / \mathrm{ml})$ and ionomycin $(1 \mu \mathrm{g} / \mathrm{ml})$ with brefeldin $\mathrm{A}(10 \mu \mathrm{g} / \mathrm{ml})$ added for the final $4 \mathrm{~h}$. Values represent the percentage of cytokine-positive cells as a percentage of the total CD4 T cell population (Ttot). Gates determining positive cells were set using equivalent non-PMA/ionomycin-stimulated controls. The percentage of positive cells for IL-17 and IFN $\gamma$ unstimulated cultures was $<1 \%$ and $<2 \%$, respectively. Data shown are representative of two independent experiments.

tolDCs ex vivo for autologous cellular immunotherapy. To our knowledge, this is the first study to demonstrate the generation of tolDCs from RA patients.

tolDCs for immunotherapy must be safe, standardised and controlled $^{3031}$; however, robust and reliable markers are poorly defined. Ideally, OC of tolDCs should be based on markers that are quickly and readily detectable. Phenotypic identification of tolDCs is most commonly determined relative to other DC populations (eg, iDCs and/or matDCs ${ }^{47}$ ), which is impractical in the cGMP laboratory setting. This study revealed the exclusive high-level expression of TLR-2 by tolDCs, a known response to glucocorticoids, ${ }^{49-51}$ making this an ideal marker for $\mathrm{QC}$ purposes.

Of particular importance in tolDC treatment of inflammatory autoimmune diseases including RA is their stability. We therefore investigated the stability of tolDCs to a number of known proinflammatory mediators in vitro, as the use of maturation-sensitive tolDCs in ongoing inflammatory disease risks the conversion of tolDCs into matDCs with the potential to exacerbate disease. ${ }^{52}$ This study revealed that cGMP-tolDCs, despite differential signalling of MPLA and
LPS via TLR $-4,{ }^{43}$ were rendered refractory to further maturation with LPS, a phenomenon previously described for LPS as LPS desensitisation. ${ }^{53}$ tolDCs were also resistant to maturation and maintained stable cytokine production in response to stimulation with proinflammatory cytokines, a process termed DC exhaustion or paralysis. ${ }^{33} 5455$ Even though the tolDCs described here express high levels of TLR-2, PGN, a microbial compound recognised by TLR-2,56 had only subtle effects on tolDCs. Consistent with our findings, it was recently shown that LPS-activated, Dex-treated DCs further challenged with LPS in the absence of Dex were phenotypically stable and remained poor producers of TNF $\alpha$ and IL- $6 .{ }^{57}$ However, other TLR ligands or proinflammatory cytokines were not investigated in that study. In contrast with the study of Chamorro et al, ${ }^{51}$ our study revealed a downregulation of TLR-2 expression in the absence of Dex, and TLR-2- or TLR-4-mediated challenge of tolDCs failed to enhance TLR-2 expression by tolDCs. This may be explained by the different TLR-2 ligands used or be due to the different protocols used for the establishment of tolDCs. Importantly, the cGMP-tolDCs described here, once removed from the 
A

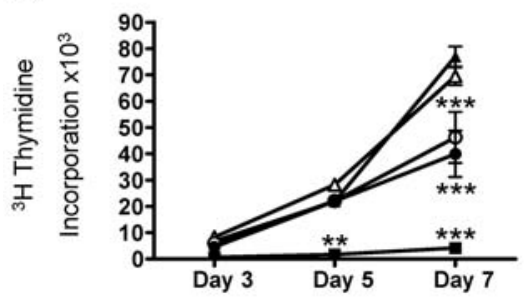

tolDC

$\Delta$ matDC

$\triangle$ matDC + matDC

matDC + tolDC $1: 1$

O matDC + tolDC 10:1

B

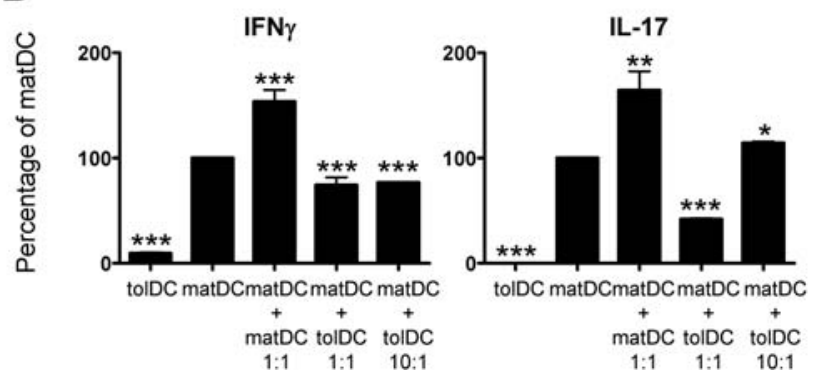

Figure 5 Tolerogenic dendritic cells (tolDCs) suppress mature DC (matDC)-induced T cell proliferation, interferon (IFN) $\gamma$ and interleukin (IL)-17. (A) Representative graph showing control CD4 T cell responses to purified protein derivative (PPD)-loaded DCs $(1 \mu \mathrm{g} / \mathrm{ml})$. CD4 T cells $\left(10^{5}\right)$ were cocultured at a 10:1 ratio with tolDCs or matDCs alone or matDCs with an equivalent number of tolDCs or matDCs as a cell number control (1:1 ratio; $\left.10^{4}\right)$ or 10-fold fewer tolDCs than matDCs $\left(1: 10\right.$ ratio; $\left.10^{3}\right)$. Proliferation was quantitated at days 3,5 and 7 by $\left[{ }^{3} \mathrm{H}\right]$ thymidine incorporation. The graph is representative of four independent experiments. (B) Mean IFN $\gamma$ and IL-17 cytokine production relative to matDCs. CD4 T cells $\left(10^{5}\right)$ were stimulated with autologous PPD-loaded DCs as in (A). Cytokine production was quantitated on day 6 by ELISA. Data are expressed as the mean \pm SEM from three independent experiments. ${ }^{*} p \leq 0.05,{ }^{* *} p \leq 0.01,{ }^{* * *} p \leq 0.001$ as compared with matDCs (two-way analysis of variance $(A)$ and analysis of variance (B)).

tolerising environment of Dex, VitD3 and MPLA, maintained a typical semimature phenotype and failed to secrete detectable IL-12 in response to a variety of DC maturation factors, making them well suited for the treatment of ongoing inflammatory disease.

A major advantage of tolDC immunotherapy is the potential for antigen-specific $T$ cell tolerisation, thereby minimising the need for sustained non-specific therapies with side effects. Our study demonstrates that tolDCs suppress immunogenic $\mathrm{DC}$-induced $\mathrm{T}$ cell proliferation at a 1:10 tolDC/matDC ratio in vitro. Clinical trial of tolDC therapy will ultimately reveal tolDC efficacy; however, localised administration of tolDCs to the arthritic joint will facilitate high tolDC/matDC ratios, which may be required for clinical efficacy and the reinduction of $T$ cell tolerance. Using model antigens, we demonstrate that tolDCs are unaffected by antigen loading and mediate antigen-specific hyporesponsiveness without inducing proliferation of 'bystander' $T$ cells with low affinity for the antigen, all of which are important features of targeted antigen-specific therapy. The pathogenic autoantigen in RA is unknown, however; future studies will reveal the tolerising effects of tolDCs to non-characterised RA autoantigen(s) present within synovial fluid. ${ }^{58-60}$

This study describes the development of clinical-grade tolDCs established using a simple and robust protocol. These tolDCs are classically semimature with an anti-inflammatory phenotype and exhibit high-level expression of TLR-2, highly
A
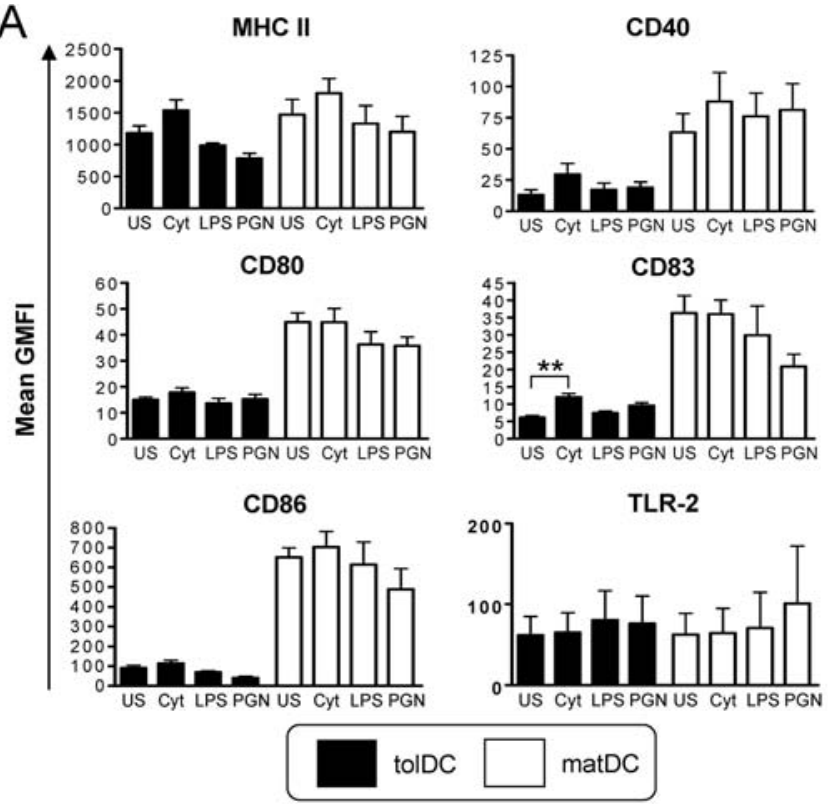

B IL-10

IL-12

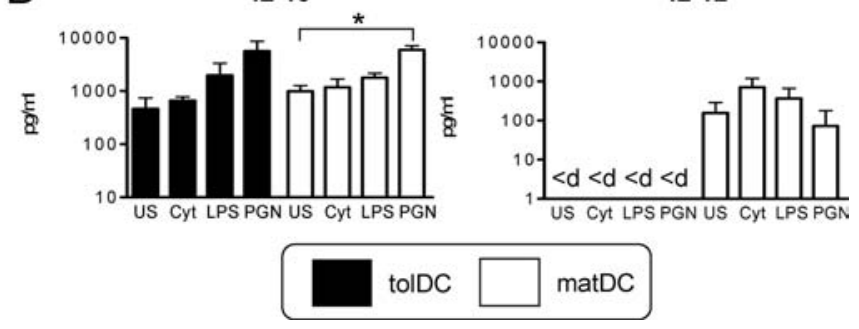

Figure 6 Tolerogenic dendritic cells (tolDCs) have a highly stable semimature, anti-inflammatory phenotype. Phenotypic stability of tolDCs and mature DCs (matDCs) was determined in response to inflammatory stimuli. tolDCs or matDCs were washed and recultured in the absence of tolerising factors including dexamethasone and vitamin $D_{3}$ for $24 \mathrm{~h}$. Cultures were either unstimulated (US) or stimulated with a cocktail of proinflammatory cytokines (cyt) containing interleukin (IL)-1 $\beta$, IL-6, tumour necrosis factor (TNF) $\alpha$ (all $10 \mathrm{ng} / \mathrm{ml}$ ) and interferon (IFN) $\gamma(1000$ $\mathrm{U} / \mathrm{ml})$, lipopolysaccharide (LPS) $(0.1 \mu \mathrm{g} / \mathrm{ml})$ or peptidoglycan (PGN) $(10 \mu \mathrm{g} / \mathrm{ml})$. (A) DCs were analysed for cell surface phenotype by flow cytometry. Plots show mean geometric mean fluorescence intensity (GMFI) of DC-maturation markers of tolDCs and matDCs. Data are expressed as the mean $\mathrm{GMFI} \pm \mathrm{SEM}$ of at least four independent donors. (B) tolDCs and matDCs (105/well) were activated via CD40 by culture with an equivalent number of the J588L-CD40L-expressing cell line in the presence or absence of inflammatory mediators and supernatants collected after $24 \mathrm{~h}$ for analysis by ELISA. $<\mathrm{d}$, below limits of detection $\left(30 \mathrm{pg} / \mathrm{ml}\right.$ for IL-12 ELISA) ${ }^{*} \mathrm{p}<0.05,{ }^{* *} \mathrm{p} \leq 0.01$ (analysis of variance). MHC, major histocompatibility complex; TLR-2, toll-like receptor 2 .

appropriate for QC. Potently tolerogenic to autologous $\mathrm{T}$ cells, these cGMP-tolDCs are highly stable in proinflammatory environments. tolDCs are a promising novel autologous cellular therapy for the specific modulation of autoreactive $T$ cells in the treatment of RA and other inflammatory autoimmune diseases.

Acknowledgements We thank Mr T Wooldridge and Dr G Raftery for the recruitment of patients with RA, and J Diboll for technical assistance. Mr T Wooldridge is funded by the UK NIHR Biomedical Research Centre for Ageing and Age-related disease award to the Newcastle upon Tyne Hospitals NHS Foundation Trust. This work was supported by the JGW Patterson Foundation and Arthritis Research UK (grant 17750). 
Funding JGW Patterson Foundation, Arthritis Research Campaign.

Competing interests None.

Ethics approval This study was conducted with the approval of the Newcastle and North Tyneside Research Ethics Committee 2.

Provenance and peer review Not commissioned; externally peer reviewed.

\section{REFERENCES}

1. Isaacs JD. T cell immunomodulation - the Holy Grail of therapeutic tolerance. Curr Opin Pharmacol 2007; 7:418-25.

2. van Duivenvoorde LM, van Mierlo GJ, Boonman ZF, et al. Dendritic cells: vehicles for tolerance induction and prevention of autoimmune diseases. Immunobiology 2006:211:627-32.

3. Banchereau J, Steinman RM. Dendritic cells and the control of immunity. Nature 1998;392:245-52

4. Ohnmacht C, Pullner A, King SB, et al. Constitutive ablation of dendritic cells breaks self-tolerance of CD4 T cells and results in spontaneous fatal autoimmunity. J Exp Med 2009;206:549-59.

5. Thomson AW, Robbins PD. Tolerogenic dendritic cells for autoimmune disease and transplantation. Ann Rheum Dis 2008;67(Suppl 3):iii90-6.

6. Jonuleit $\mathbf{H}$, Schmitt E, Steinbrink K, et al. Dendritic cells as a tool to induce anergic and regulatory T cells. Trends Immunol 2001;22:394-400.

7. Coates PT, Colvin BL, Kaneko K, et al. Pharmacologic, biologic, and genetic engineering approaches to potentiation of donor-derived dendritic cell tolerogenicity. Transplantation 2003;75(9 Suppl):32S-6S.

8. Morita Y, Yang J, Gupta R, et al. Dendritic cells genetically engineered to express IL-4 inhibit murine collagen-induced arthritis. J Clin Invest 2001;107:1275-84.

9. Kim SH, Kim S, Evans CH, et al. Effective treatment of established murine collageninduced arthritis by systemic administration of dendritic cells genetically modified to express IL-4. J Immunol 2001;166:3499-505

10. Steinbrink K, WölfI M, Jonuleit $\mathrm{H}$, et al. Induction of tolerance by IL-10-treated dendritic cells. J Immunol 1997;159:4772-80.

11. van Duivenvoorde LM, Han WG, Bakker AM, et al. Immunomodulatory dendritic cells inhibit Th1 responses and arthritis via different mechanisms. J Immunol 2007:179:1506-15

12. van Duivenvoorde LM, Louis-Plence P, Apparailly F, et al. Antigen-specific immunomodulation of collagen-induced arthritis with tumor necrosis factor-stimulated dendritic cells. Arthritis Rheum 2004;50:3354-64.

13. Healy LJ, Collins HL, Thompson SJ. Systemic administration of tolerogenic dendritic cells ameliorates murine inflammatory arthritis. Open Rheumatol $J$ 2008;2:71-80.

14. Bianco NR, Kim SH, Ruffner MA, et al. Therapeutic effect of exosomes from indoleamine 2,3-dioxygenase-positive dendritic cells in collagen-induced arthritis and delayed-type hypersensitivity disease models. Arthritis Rheum 2009:60:380-9.

15. Jaen 0, Rullé $S$, Bessis N, et al. Dendritic cells modulated by innate immunity improve collagen-induced arthritis and induce regulatory T cells in vivo. Immunology 2009;126:35-44.

16. Chorny A, Gonzalez-Rey E, Fernandez-Martin A, et al. Vasoactive intestinal peptide induces regulatory dendritic cells with therapeutic effects on autoimmune disorders. Proc Natl Acad Sci USA 2005;102:13562-7.

17. Salazar L, Aravena 0, Abello P, et al. Modulation of established murine collageninduced arthritis by a single inoculation of short-term lipopolysaccharide-stimulated dendritic cells. Ann Rheum Dis 2008;67:1235-41.

18. Popov I, Li M, Zheng X, et al. Preventing autoimmune arthritis using antigenspecific immature dendritic cells: a novel tolerogenic vaccine. Arthritis Res Ther 2006;: : R141

19. Adorini L. Tolerogenic dendritic cells induced by vitamin $D$ receptor ligands enhance regulatory T cells inhibiting autoimmune diabetes. Ann N Y Acad Sci 2003;987:258-61.

20. Lau AW, Biester S, Cornall RJ, et al. Lipopolysaccharide-activated IL-10secreting dendritic cells suppress experimental autoimmune uveoretinitis by MHCIl-dependent activation of CD62L-expressing regulatory T cells. J Immunol 2008; 180:3889-99

21. Peng JC, Thomas R, Nielsen LK. Generation and maturation of dendritic cells for clinical application under serum-free conditions. J Immunother 2005;28:599-609.

22. de Vries IJ, Eggert AA, Scharenborg NM, et al. Phenotypical and functional characterization of clinical grade dendritic cells. J Immunother 2002;25:429-38.

23. Napoletano C, Pinto D, Bellati F, et al. A comparative analysis of serum and serumfree media for generation of clinical grade DCs. J Immunother 2007;30:567-76.

24. Anderson AE, Sayers BL, Haniffa MA, et al. Differential regulation of naïve and memory CD4 + T cells by alternatively activated dendritic cells. J Leukoc Biol 2008;84:124-33.

25. Anderson AE, Swan DJ, Sayers BL, et al. LPS activation is required for migratory activity and antigen presentation by tolerogenic dendritic cells. J Leukoc Biol 2009:85:243-50.

26. Hilkens CM, Isaacs JD, Thomson AW. Development of dendritic cell-based immunotherapy for autoimmunity. Int Rev Immunol 2010;29:156-83.
27. Hilkens CM, Isaacs JD. Tolerogenic dendritic cells in clinical practice. Open Arthritis Journal 2010;3:8-12.

28. Radstake TR, van Lent PL, Pesman GJ, et al. High production of proinflammatory and Th1 cytokines by dendritic cells from patients with rheumatoid arthritis, and down regulation upon FcgammaR triggering. Ann Rheum Dis 2004;63:696-702.

29. Radstake TR, Blom AB, Slöetjes AW, et al. Increased FcgammaRIl expression and aberrant tumour necrosis factor alpha production by mature dendritic cells from patients with active rheumatoid arthritis. Ann Rheum Dis 2004;63:1556-63.

30. Figdor CG, de Vries IJ, Lesterhuis WJ, et al. Dendritic cell immunotherapy: mapping the way. Nat Med 2004;10:475-80.

31. Nestle FO, Banchereau J, Hart D. Dendritic cells: on the move from bench to bedside Nat Med 2001;7:761-5

32. Langenkamp A, Casorati G, Garavaglia C, et al. T cell priming by dendritic cells: thresholds for proliferation, differentiation and death and intraclonal functional diversification. Eur J Immunol 2002;32:2046-54.

33. Kalinski P, Schuitemaker JH, Hilkens CM, et al. Final maturation of dendritic cells is associated with impaired responsiveness to IFN-gamma and to bacterial IL-12 inducers: decreased ability of mature dendritic cells to produce IL-12 during the interaction with Th cells. J Immunol 1999;162:3231-6.

34. Hilkens $\mathbf{C M}$, Kalinski $\mathrm{P}$, de Boer $\mathrm{M}$, et al. Human dendritic cells require exogenous interleukin-12-inducing factors to direct the development of naive T-helper cells toward the Th1 phenotype. Blood 1997;90:1920-6.

35. Mandron M, Ariès MF, Brehm RD, et al. Human dendritic cells conditioned with Staphylococcus aureus enterotoxin B promote TH2 cell polarization. J Allergy Clin Immunol 2006;117:1141-7.

36. Melkus MW, Estes JD, Padgett-Thomas A, et al. Humanized mice mount specific adaptive and innate immune responses to EBV and TSST-1. Nat Med 2006;12:1316-22.

37. Mingari MC, Cambiaggi A, Vitale $\mathrm{C}$, et al. Effect of superantigens on human thymocytes: selective proliferation of $\mathrm{V}$ beta $2+$ cells in response to toxic shock syndrome toxin-1 and their deletion upon secondary stimulation. Int Immunol 1996;8:203-9.

38. Ovali E, Dikmen T, Sonmez $\mathrm{M}$, et al. Active immunotherapy for cancer patients using tumor lysate pulsed dendritic cell vaccine: a safety study. J Exp Clin Cancer Res 2007:26:209-14.

39. Curti A, Tosi P, Comoli P, et al. Phase I/II clinical trial of sequential subcutaneous and intravenous delivery of dendritic cell vaccination for refractory multiple myeloma using patient-specific tumour idiotype protein or idiotype (VDJ)-derived class I-restricted peptides. Br J Haematol 2007;139:415-24.

40. Motta MR, Castellani S, Rizzi S, et al. Generation of dendritic cells from CD14+ monocytes positively selected by immunomagnetic adsorption for multiple myeloma patients enrolled in a clinical trial of anti-idiotype vaccination. Br J Haematol 2003;121:240-50

41. Makkouk A, Abdelnoor AM. The potential use of Toll-like receptor (TLR) agonists and antagonists as prophylactic and/or therapeutic agents. Immunopharmacol Immunotoxicol 2009:31:331-8.

42. Ismaili J, Rennesson J, Aksoy E, et al. Monophosphoryl lipid A activates both human dendritic cells and T cells. J Immunol 2002;168:926-32.

43. Mata-Haro V, Cekic C, Martin M, et al. The vaccine adjuvant monophosphoryl lipid A as a TRIF-biased agonist of TLR4. Science 2007:316:1628-32.

44. Henricson BE, Manthey CL, Perera PY, et al. Dissociation of lipopolysaccharide (LPS)inducible gene expression in murine macrophages pretreated with smooth LPS versus monophosphoryl lipid A. Infect Immun 1993;61:2325-33.

45. Salkowski CA, Detore GR, Vogel SN. Lipopolysaccharide and monophosphoryl lipid A differentially regulate interleukin-12, gamma interferon, and interleukin-10 mRNA production in murine macrophages. Infect Immun 1997;65:3239-47.

46. Martin M, Michalek SM, Katz J. Role of innate immune factors in the adjuvant activity of monophosphoryl lipid A. Infect Immun 2003;71:2498-507.

47. Steinman RM, Hawiger D, Nussenzweig MC. Tolerogenic dendritic cells. Annu Rev Immunol 2003;21:685-711.

48. Rutella S, De Cristofaro R, Ferraccioli G. Function and dysfunction of dendritic cells in autoimmune rheumatic diseases. Hum Immunol 2009:70:360-73.

49. Rozkova D, Horvath R, Bartunkova J, et al. Glucocorticoids severely impair differentiation and antigen presenting function of dendritic cells despite upregulation of Toll-like receptors. Clin Immuno/ 2006;120:260-71.

50. Shibata M, Katsuyama M, Onodera T, et al. Glucocorticoids enhance Toll-like receptor 2 expression in human keratinocytes stimulated with Propionibacterium acnes or proinflammatory cytokines. J Invest Dermatol 2009;129:375-82.

51. Chamorro S, García-Vallejo JJ, Unger WW, et al. TLR triggering on tolerogenic dendritic cells results in TLR2 up-regulation and a reduced proinflammatory immune program. J Immuno/ 2009;183:2984-94.

52. Voigtländer C, Rössner S, Cierpka E, et al. Dendritic cells matured with TNF can be further activated in vitro and after subcutaneous injection in vivo which converts their tolerogenicity into immunogenicity. J Immunother 2006;29:407-15.

53. Dobrovolskaia MA, Vogel SN. Toll receptors, CD14, and macrophage activation and deactivation by LPS. Microbes Infect 2002;4:903-14.

54. Langenkamp A, Messi M, Lanzavecchia A, et al. Kinetics of dendritic cell activation: impact on priming of TH1, TH2 and nonpolarized T cells. Nat Immunol 2000:1:311-16 
55. Reis e Sousa C, Yap G, Schulz 0, et al. Paralysis of dendritic cell IL-12 production by microbial products prevents infection-induced immunopathology. Immunity 1999:11:637-47.

56. Asong J, Wolfert MA, Maiti KK, et al. Binding and cellular activation studies reveal that toll-like receptor 2 can differentially recognize peptidoglycan from gram-positive and gram-negative bacteria. J Biol Chem 2009;284:8643-53.

57. Bosma BM, Metselaar HJ, Nagtzaam NM, et al. Dexamethasone transforms lipopolysaccharide-stimulated human blood myeloid dendritic cells into myeloid dendritic cells that prime interleukin-10 production in T cells. Immunology 2008:125:91-100.
58. Tsark EC, Wang W, Teng YC, et al. Differential MHC class II-mediated presentation of rheumatoid arthritis autoantigens by human dendritic cells and macrophages. $J$ Immunol 2002;169:6625-33.

59. Tran CN, Davis MJ, Tesmer LA, et al. Presentation of arthritogenic peptide to antigen-specific T cells by fibroblast-like synoviocytes. Arthritis Rheum 2007:56:1497-506

60. van Lierop MJ, den Hoed L, Houbiers J, et al. Endogenous HLA-DRrestricted presentation of the cartilage antigens human cartilage gp-39 and melanoma inhibitory activity in the inflamed rheumatoid joint. Arthritis Rheum 2007:56:2150-9. 